\title{
Fuzzy Ideals and Fuzzy Filters of Ordered Ternary Semigroups
}

\author{
Ronnason Chinram (Corresponding author) \\ Department of Mathematics, Prince of Songkla University \\ Hat Yai, Songkhla, Thailand \\ Tel: 66-89-731-3988 E-mail: ronnason.c@psu.ac.th \\ Sompob Saelee \\ Department of Mathematics, Prince of Songkla University \\ Hat Yai, Songkhla, Thailand \\ Tel: 66-83-512-5787 E-mail: pob_lee@ hotmail.com
}

\begin{abstract}
The notion of ternary semigroups was introduced by Lehmer in 1932 and that of fuzzy sets by Zadeh in 1965 . Any semigroup can be reduced to a ternary semigroup but a ternary semigroup does not necessarily reduce to a semigroup. A partially ordered semigroup $T$ is called an ordered ternary semigroup if for all $x_{1}, x_{2}, x_{3}, x_{4} \in T, x_{1} \leq x_{2}$ implies $x_{1} x_{3} x_{4} \leq x_{2} x_{3} x_{4}, x_{3} x_{1} x_{4} \leq x_{3} x_{2} x_{4}$ and $x_{3} x_{4} x_{1} \leq x_{3} x_{4} x_{2}$. In this paper, we study fuzzy ternary subsemigroups (left ideals, right ideals, lateral ideals, ideals) and fuzzy left filters (right filters, lateral filters, filters) of ordered ternary semigroups.
\end{abstract}

Keywords: Fuzzy ideals, Prime fuzzy ideals, Filters, Fuzzy filters, Ordered ternary semigroups

\section{Introduction and Preliminaries}

In 1932, Lehmer gave the definition of ternary semigroups (Lehmer, 1932). A nonempty set $T$ is called a ternary semigroup if there exists a ternary operation $T \times T \times T \rightarrow T$, written as $\left(x_{1}, x_{2}, x_{3}\right) \mapsto x_{1} x_{2} x_{3}$ satisfying the following identity for any $x_{1}, x_{2}, x_{3}, x_{4}, x_{5} \in T$,

$$
\left[\left[x_{1} x_{2} x_{3}\right] x_{4} x_{5}\right]=\left[x_{1}\left[x_{2} x_{3} x_{4}\right] x_{5}\right]=\left[x_{1} x_{2}\left[x_{3} x_{4} x_{5}\right]\right]
$$

Any semigroup can be reduced to a ternary semigroup. However, Banach showed that a ternary semigroup does not necessarily reduce to a semigroup by this example.

Example 1.1 $T=\{-i, 0, i\}$ is a ternary semigroup while $T$ is not a semigroup under the multiplication over complex numbers.

The next example is also a ternary semigroup but not a semigroup.

Example $1.2 \mathbb{Z}^{-}$is a ternary semigroup while $\mathbb{Z}^{-}$is not a semigroup under the multiplication over integers.

However, Los showed that every ternary semigroup can be embedded in a semigroup (Los, 1955).

The algebraic structures of ternary semigroups were studied by some authors, for example, Sioson studied ideals in ternary semigroups (Sioson, 1965), Santiago studied regular ternary semigroups (Santiago, 1990), Dixit and Dewan studied quasi-ideals and bi-ideals in ternary semigroups (Dixit and Dewan, 1995), Kar and Maity studied congruences of ternary semigroups (Kar and Maity, 2007) and Iampan studied minimal and maximal lateral ideals of ternary semigroups (Iampan, 2007).

A partially ordered semigroup $T$ is called an ordered ternary semigroup if for all $x_{1}, x_{2}, x_{3}, x_{4} \in T, x_{1} \leq x_{2}$ implies $x_{1} x_{3} x_{4} \leq x_{2} x_{3} x_{4}, x_{3} x_{1} x_{4} \leq x_{3} x_{2} x_{4}$ and $x_{3} x_{4} x_{1} \leq x_{3} x_{4} x_{2}$.

Example $1.3\left(\mathbb{Z}^{-}, \cdot, \leq\right)$ is a ordered ternary semigroup.

Let $T$ be an ordered ternary semigroup. For nonempty subsets $A, B$ and $C$ of $T$, let $A B C:=\{a b c \mid a \in A, b \in B$ and $c \in C\}$. For a nonempty subset $A$ of $T$, we note $(A]:=\{t \in T \mid t \leq h$ for some $h \in A\}$. A nonempty subset $S$ of $T$ is called a ternary subsemigroup of $T$ if $(S] \subseteq S$ and $S S S \subseteq S$. A nonempty subset $A$ of $T$ is called a left ideal of $T$ if $(A] \subseteq A$ and $T T A \subseteq A$, a right ideal of $T$ if $(A] \subseteq A$ and $A T T \subseteq T$, and a lateral ideal of $T$ if $(A] \subseteq A$ and $T A T \subseteq A$. If $A$ is a left, right and lateral ideal of $T, A$ is called an ideal of $T$. 
The notion of fuzzy sets was introduced by Zadeh (Zadeh, 1965). Several researchs were conducted on the generalizations of the notion of fuzzy sets. Fuzzy semigroups have been first considered by Kuroki (Kuroki, 1981, 1991 and 1993) and fuzzy ordered semigroups by Kehayopulu and Tsingelis (Kehayopulu, 1990, Kehayopulu and Tsingelis, 1999 and 2002 , Kehayopulu, Xie and Tsingelis, 2001). In 2008, Shabir and Khan studied fuzzy filters in ordered semigroups (Shabir and Khan, 2008).

Let $T$ be an ordered ternary semigroup. A function $f$ from $T$ to the unit interval $[0,1]$ is called a fuzzy subset of $T$. The ordered ternary semigroup $T$ itself is a fuzzy subset of $T$ such that $T(x)=1$ for all $x \in T$, denoted also by $T$. If $A \subseteq T$, the characteristic function $f_{A}$ of $A$ is a fuzzy subset of $T$ defined as follows:

$$
f_{A}(x)= \begin{cases}1 & \text { if } x \in A, \\ 0 & \text { if } x \notin A .\end{cases}
$$

Let $T$ be an ordered ternary semigroup and $f$ a fuzzy subset of $T$. The fuzzy subset $f^{\prime}$ defined by $f^{\prime}(x)=1-f(x)$ for all $x \in T$ is called the complement of $f$ in $T$.

The aim of this paper is to study fuzzy ternary subsemigroups (left ideals, right ideals, lateral ideals, ideals) and fuzzy left filters (right filters, lateral filters, filters) of ordered ternary semigroups that are studied analogously to the concept of fuzzy ideals and fuzzy filters in ordered semigroups.

\section{Main Results}

Now we define fuzzy ternary subsemigroups, fuzzy left ideals, fuzzy right ideals, fuzzy lateral ideals and fuzzy ideals of ordered ternary semigroups. Let $T$ be a ternary semigroup. A fuzzy subset $f$ of $T$ is called

a fuzzy ternary subsemigroup of $T$ if (1) $x \leq y$ implies $f(x) \geq f(y)$ and (2) $f(x y z) \geq \min \{f(x), f(y), f(z)\}$ for all $x, y, z \in T$, a fuzzy left ideal of $T$ if (1) $x \leq y$ implies $f(x) \geq f(y)$ and (2) $f(x y z) \geq f(z)$ for all $x, y, z \in T$, a fuzzy right ideal of $T$ if (1) $x \leq y$ implies $f(x) \geq f(y)$ and (2) $f(x y z) \geq f(x)$ for all $x, y, z \in T$, a fuzzy lateral ideal of $T$ if (1) $x \leq y$ implies $f(x) \geq f(y)$ and (2) $f(x y z) \geq f(y)$ for all $x, y, z \in T$ and a fuzzy ideal of $T$ if (1) $x \leq y$ implies $f(x) \geq f(y)$ and (2) $f(x y z) \geq \max \{f(x), f(y), f(z)\}$ for all $x, y, z \in T$.

Lemma 2.1 Let $T$ be an ordered ternary semigroup and $A$ a nonempty subset of $T$. Then $(A] \subseteq A$ if and only if $x \leq y$ implies $f_{A}(x) \geq f_{A}(y)$.

Proof Assume that $(A] \subseteq A$. Let $x, y \in T$ such that $x \leq y$.

Case 1: $y \notin A$. Then $f_{A}(y)=0 \leq f_{A}(x)$.

Case 2: $y \in A$. Since $(A] \subseteq A, x \in A$. So $f_{A}(x)=1 \geq f_{A}(y)$.

Conversely, let $x \in(A]$. Then there exists $y \in A$ such that $x \leq y$. So $f_{A}(x) \geq f_{A}(y)=1$. This implies $x \in A$.

Now we characterize ternary subsemigroups (left ideals, right ideals, lateral ideals, ideals) of ordered ternary semigroups in terms of fuzzy ternary subsemigroups (fuzzy left ideals, fuzzy right ideals, fuzzy lateral ideals, fuzzy ideals).

Theorem 2.1 Let $T$ be an ordered ternary semigroup and $A$ a nonempty subset of $T$. The following statements are true.

(1) $A$ is a ternary subsemigroup of $T$ if and only if $f_{A}$ is a fuzzy ternary subsemigroup of $T$.

(2) $A$ is a left ideal (right ideal, lateral ideal, ideal) of $T$ if and only if $f_{A}$ is a fuzzy left ideal (fuzzy right ideal, fuzzy lateral ideal, fuzzy ideal) of $T$.

Proof (1) Assume that $A$ is a ternary subsemigroup of $T$. By Lemma 2.1, $x \leq y$ implies $f_{A}(x) \geq f_{A}(y)$. Next, let $x, y, z \in T$.

Case $1: x, y, z \in A$. Since $A$ is a ternary subsemigroup of $T, x y z \in A$. Therefore $f_{A}(x y z)=1 \geq \min \{f(x), f(y), f(z)\}$.

Case 2: $x \notin A$ or $y \notin A$ or $z \notin A$. Thus $f_{A}(x)=0$ or $f_{A}(y)=0$ or $f_{A}(z)=0$. Hence $\min \left\{f_{A}(x), f_{A}(y), f_{A}(z)\right\}=0 \leq f_{A}(x y z)$.

Conversely, assume that $f_{A}$ is a fuzzy ternary subsemigroup of $T$. By Lemma 2.1, $(A] \subseteq A$. Next, let $x, y, z \in A$. So $f_{A}(x)=f_{A}(y)=f_{A}(z)=1$. Since $f_{A}$ is a fuzzy ternary subsemigroup of $T, f_{A}(x y z) \geq \min \left\{f_{A}(x), f_{A}(y), f_{A}(z)\right\}=1$. Then $x y z \in A$.

(2) Assume that $A$ is a left ideal of $T$. By Lemma 2.1, we have that $x \leq y$ implies $f_{A}(x) \geq f_{A}(y)$. Next, let $x, y, z \in T$.

Case $1: z \in A$. Since $A$ is a left ideal of $T, x y z \in A$. Then $f_{A}(x y z)=1$. Therefore $f_{A}(x y z) \geq f_{A}(z)$.

Case $2: z \notin A$. So $f_{A}(z)=0$. Hence $f_{A}(x y z) \geq f_{A}(z)$.

Conversely, assume that $f_{A}$ is a fuzzy left ideal of $T$. By Lemma 2.1, $(A] \subseteq A$. Next, let $x, y \in T$ and $z \in A$. Since $f_{A}$ is a fuzzy left ideal of $T$ and $z \in A, f_{A}(x y z) \geq f_{A}(z)=1$. So $x y z \in A$.

The other parts of (2) can be proved in similarly way. 
Now we define left filters, right filters, lateral filters, filters, fuzzy left filters, fuzzy right filters, fuzzy lateral filters and fuzzy filters of ordered ternary semigroups.

Let $T$ be an ordered ternary semigroup. A nonempty subset $F$ of $T$ is called

a left filter of $T$ if (1) $F^{3} \subseteq F$, (2) for all $x, y \in T, x \leq y$ and $x \in F$ imply $y \in F$ and (3) for all $x, y, z \in T, x y z \in F$ implies $z \in F$,

a right filter of $T$ if (1) $F^{3} \subseteq F$, (2) for all $x, y \in T, x \leq y$ and $x \in F$ imply $y \in F$ and (3) for all $x, y, z \in T, x y z \in F$ implies $y \in F$,

a lateral filter of $T$ if (1) $F^{3} \subseteq F$, (2) for all $x, y \in T, x \leq y$ and $x \in F$ imply $y \in F$ and (3) for all $x, y, z \in T, x y z \in F$ implies $x \in F$ and

a filter of $T$ if (1) $F^{3} \subseteq F$, (2) for all $x, y \in T, x \leq y$ and $x \in F$ imply $y \in F$ and (3) for all $x, y, z \in T, x y z \in F$ implies $x, y, z \in F$.

A fuzzy subset $f$ of $T$ is called

a fuzzy left filter of $T$ if for all $x, y, z \in T(1) x \leq y$ implies $f(x) \leq f(y),(2) f(x y z) \geq \min \{f(x), f(y), f(z)\}$ and $(3) f(x y z) \leq$ $f(z)$

a fuzzy right filter of $T$ if for all $x, y, z \in T$ (1) $x \leq y$ implies $f(x) \leq f(y)$, (2) $f(x y z) \geq \min \{f(x), f(y), f(z)\}$ and (3) $f(x y z) \leq f(x)$,

a fuzzy lateral filter of $T$ if for all $x, y, z \in T$ (1) $x \leq y$ implies $f(x) \leq f(y)$, (2) $f(x y z) \geq \min \{f(x), f(y), f(z)\}$ and (3) $f(x y z) \leq f(y)$ and

a fuzzy filter of $T$ if for all $x, y, z \in T(1) x \leq y$ implies $f(x) \leq f(y),(2) f(x y z)=\min \{f(x), f(y), f(z)\}$.

We also characterize left filters (right filters, lateral filters, filters) of ordered ternary semigroups in terms of fuzzy left filters (fuzzy right filters, fuzzy lateral filters, fuzzy filters).

Theorem 2.2 Let $F$ be a nonempty subset of an ordered ternary semigroup $T$. Then $F$ is a left filter (right filter, lateral filter, filter) of $T$ if and only if the characteristic function $f_{F}$ of $F$ is a fuzzy left filter (right filter, lateral filter, filter) of $T$.

Proof Assume that $F$ is a left filter of $T$. Let $x, y \in T$ such that $x \leq y$.

Case 1: $x \notin F$. Then $f_{F}(x)=0$. Then $f_{F}(x) \leq f_{F}(y)$.

Case 2: $x \in F$. Since $x \leq y$ and $F$ is a left filter of $T, y \in F$. Thus $f_{F}(y)=1$. Hence $f_{F}(x) \leq f_{F}(y)$.

Next, let $x, y, z \in T$.

Case 1: $x, y, z \in F$. Then $x y z \in F$. Hence $f_{F}(x y z)=1$. Therefore $f_{F}(x y z) \geq \min \left\{f_{F}(x), f_{F}(y), f_{F}(z)\right\}$.

Case 2: $x \notin F$ or $y \notin F$ or $z \notin F$. So $f_{F}(x)=0$ or $f_{F}(y)=0$ or $f_{F}(z)=0$. This implies $f_{F}(x y z) \geq \min \left\{f_{F}(x), f_{F}(y), f_{F}(z)\right\}$.

Finally, let $x, y, z \in T$.

Case 1: $x y z \in F$. Since $F$ is a left filter of $T$ and $x y z \in F, z \in F$. So $f_{F}(z)=1$. Therefore $f_{F}(x y z) \leq f_{F}(z)$.

Case 2: $x y z \notin F$. Then $f_{F}(x y z)=0$. Therefore $f_{F}(x y z) \leq f_{F}(z)$.

Conversely, assume $f_{F}$ is a fuzzy left filter of $T$. Let $x, y, z \in F$. Then $f_{F}(x)=f_{F}(y)=f_{F}(z)=1$. Thus $f_{F}(x y z) \geq$ $\min \left\{f_{F}(x), f_{F}(y), f_{F}(z)\right\}=1$. Hence $x y z \in F$. Next, let $x, y \in T$. Assume $x \leq y$ and $x \in F$. Then $f_{F}(x) \leq f_{F}(y)$ and $f_{F}(x)=1$. Thus $f_{F}(y)=1$, this implies $y \in F$. Finally, let $x, y, z \in T$ such that $x y z \in F$. So $f_{F}(x y z)=1$. Since $f_{F}$ is a fuzzy left filter of $T$, then $f_{F}(z) \geq f_{F}(x y z)$. This implies $f_{F}(z)=1$. So $z \in F$.

The other parts can be proved in similarly way.

Let $T$ be an ordered ternary semigroup. A nonempty subset $S$ of $T$ is called a prime subset of $T$ if for all $x, y, z \in T, x y z \in S$ implies $x \in S$ or $y \in S$ or $z \in S$. A ternary subsemigroup $S$ of $T$ is called a prime ternary subsemigroup of $T$ if $S$ is a prime subset of $T$. Prime left ideals, prime right ideals, prime lateral ideals and prime ideals of $T$ are defined analogously. A fuzzy subset $f$ of $T$ is called a prime fuzzy subset of $T$ if $f(x y z) \leq \max \{f(x), f(y), f(z)\}$ for all $x, y, z \in T$. A fuzzy ternary subsemigroup $f$ of $T$ is called a prime fuzzy ternary subsemigroup of $T$ if $f$ is a prime fuzzy subset of $T$. Prime fuzzy left ideals, prime fuzzy right ideals, prime fuzzy lateral ideals and prime fuzzy ideals of $T$ are defined analogously.

Theorem 2.3 Let $T$ be an ordered ternary semigroup and $A$ a nonempty subset of $T$. The following statements are true.

(1) $A$ is a prime subset of $T$ if and only if $f_{A}$ is a prime fuzzy subset of $T$.

(2) $A$ is a prime ternary subsemigroup (prime left ideal, prime right ideal, prime lateral ideal, prime ideal) of $T$ if and only if $f_{A}$ is a prime fuzzy ternary subsemigroup (prime fuzzy left ideal, prime fuzzy right ideal, prime fuzzy lateral ideal, prime fuzzy ideal) of $T$. 
Proof (1) Let $A$ be a prime subset of $T$ and $x, y, z \in T$.

Case 1: $x y z \in A$. Since $A$ is a prime subset of $T, x \in A$ or $y \in A$ or $z \in A$. So $\max \left\{f_{A}(x), f_{A}(y), f_{A}(z)\right\}=1 \geq f_{A}(x y z)$.

Case $2: x y z \notin A$. So $f_{A}(x y z)=0 \leq \max \left\{f_{A}(x), f_{A}(y), f_{A}(z)\right\}$.

Conversely, let $x, y, z \in T$ such that $x y z \in A$. Thus $f_{A}(x y z)=1$. Since $f_{A}$ is prime, $\max \left\{f_{A}(x), f_{A}(y), f_{A}(z)\right\}=1$. Then $f_{A}(x)=1$ or $f_{A}(y)=1$ or $f_{A}(z)=1$. Hence $x \in A$ or $y \in A$ or $z \in A$.

(2) follows from (1) and Theorem 2.1.

Let $f$ be a fuzzy subset of an ordered ternary semigroup $T$. For any $t \in[0,1]$, the set

$$
f_{t}=\{x \in T \mid f(x) \geq t\} \text { and } f_{t}^{s}=\{x \in T \mid f(x)>t\}
$$

are called a $t$-levelset and a $t$-strong levelset of $f$, respectively.

Theorem 2.4 Let $f$ be a fuzzy subset of an ordered ternary semigroup $T$. The following statements are true.

(1) $f$ is a fuzzy ternary subsemigroup of $T$ if and only if for all $t \in[0,1]$, if $f_{t} \neq \emptyset$, then $f_{t}$ is a ternary subsemigroup of $T$.

(2) $f$ is a fuzzy left ideal (fuzzy right ideal, fuzzy lateral ideal, fuzzy ideal) of $T$ if and only if for all $t \in[0,1]$, if $f_{t} \neq \emptyset$, then $f_{t}$ is a left ideal (right ideal, lateral ideal, ideal) of $T$.

Proof (1) Assume that $f$ is a fuzzy ternary subsemigroup of $T$. Let $t \in[0,1]$ such that $f_{t} \neq \emptyset$. Let $x \in\left(f_{t}\right]$. Then there exists $y \in f_{t}$ such that $x \leq y$. Thus $f(x) \geq f(y) \geq t$. Hence $x \in f_{t}$. Next, let $x, y, z \in f_{t}$. Then $f(x), f(y), f(z) \geq t$. Thus $\min \{f(x), f(y), f(z)\} \geq t$. Since $f$ is a fuzzy ternary subsemigroup of $T, f(x y z) \geq t$. Hence $x y z \in f_{t}$.

Conversely, assume for all $t \in[0,1]$, if $f_{t} \neq \emptyset$, then $f_{t}$ is a ternary subsemigroup of $T$. Let $x, y \in T$ such that $x \leq y$. Choose $t=f(y)$. Thus $y \in f_{t}$. This implies $x \in f_{t}$. Then $f(x) \geq t=f(y)$. Next, let $x, y, z \in T$. Choose $t=\min \{f(x), f(y), f(z)\}$. Then $f(x), f(y), f(z) \geq t$. Thus $x, y, z \in f_{t}$. Since $f_{t}$ is a ternary subsemigroup of $T, x y z \in f_{t}$. Therefore $f(x y z) \geq t=$ $\min \{f(x), f(y), f(z)\}$.

(2) Assume that $f$ is a fuzzy left ideal of $T$. Let $t \in[0,1]$. Suppose that $f_{t} \neq \emptyset$. Let $x \in\left(f_{t}\right]$. Then there exists $y \in f_{t}$ such that $x \leq y$. Thus $f(x) \geq f(y) \geq t$. Next, let $x, y, z \in T$ and $z \in f_{t}$. So $f(x y z) \geq f(z) \geq t$. Therefore $x y z \in f_{t}$.

Conversely, assume for all $t \in[0,1]$, if $f_{t} \neq \emptyset$, then $f_{t}$ is a left ideal of $T$. Let $x, y \in T$ such that $x \leq y$. Choose $t=f(y)$. Thus $y \in f_{t}$. This implies $x \in f_{t}$. Then $f(x) \geq t=f(y)$. Next, let $x, y, z \in T$. Choose $t=f(z)$. Thus $z \in f_{t}$, this implies $f_{t} \neq \emptyset$. By assumption, we have $f_{t}$ is a left ideal of $T$. So $x y z \in f_{t}$. Therefore $f(x y z) \geq t$. So $f(x y z) \geq f(z)$.

The other parts of (2) can be proved in a similar way.

Theorem 2.5 Let $f$ be a fuzzy subset of an ordered ternary semigroup $T$. The following statements are true.

(1) $f$ is a prime fuzzy subset of $T$ if and only if for all $t \in[0,1]$, if $f_{t} \neq \emptyset$, then $f_{t}$ is a prime subset of $T$.

(2) $f$ is a prime fuzzy ternary subsemigroup (prime fuzzy left ideal, prime fuzzy right ideal, prime fuzzy lateral ideal, prime fuzzy ideal) of $T$ if and only if for all $t \in[0,1]$, if $f_{t} \neq \emptyset$, then $f_{t}$ is a prime ternary subsemigroup (prime left ideal, prime right ideal, prime lateral ideal, prime ideal) of $T$.

Proof (1) Assume that $f$ is a prime fuzzy subset of $T$. Let $t \in[0,1]$. Suppose that $f_{t} \neq \emptyset$. Let $x, y, z \in T$ such that $x y z \in f_{t}$. Thus $f(x y z) \geq t$. Since $f$ is prime, $f(x) \geq t$ or $f(y) \geq t$ or $f(z) \geq t$. Hence $x \in f_{t}$ or $y \in f_{t}$ or $z \in f_{t}$.

Conversely, let $x, y, z \in T$. Choose $t=f(x y z)$. Thus $x y z \in f_{t}$. Since $f_{t}$ is prime, $x \in f_{t}$ or $y \in f_{t}$ or $z \in f_{t}$. Then $f(x) \geq t$ or $f(y) \geq t$ or $f(z) \geq t$. Therefore $\max \{f(x), f(y), f(z)\} \geq t=f(x y z)$.

(2) follows from (1) and Theorem 2.4.

Theorem 2.6 Let $f$ be a fuzzy subset of an ordered ternary semigroup $T$. Then $f$ is a fuzzy ternary subsemigroup (fuzzy left ideal, fuzzy right ideal, fuzzy lateral ideal, fuzzy ideal) of $T$ if and only if for all $t \in[0,1]$, if $f_{t}^{s} \neq \emptyset$, then $f_{t}^{s}$ is a ternary subsemigroup (left ideal, right ideal, lateral ideal, ideal) of $T$.

Proof The proof of this theorem is similar to the proof of Theorem 2.4.

Theorem 2.7 Let $f$ be a fuzzy subset of an ordered ternary semigroup $T$. Then $f$ is a prime fuzzy subset (prime fuzzy ternary subsemigroup, prime fuzzy left ideal, prime fuzzy right ideal, prime fuzzy lateral ideal, prime fuzzy ideal) of $T$ if and only if for all $t \in[0,1]$, if $f_{t}^{s} \neq \emptyset$, then $f_{t}^{s}$ is a prime subset (prime ternary subsemigroup, prime left ideal, prime right ideal, prime lateral ideal, prime ideal) of $T$.

Proof The proof of this theorem is similar to the proof of Theorem 2.5.

Lemma 2.2 Let $f$ be a fuzzy subset of an ordered ternary semigroup $T$. The following statements are equivalent.

(1) $f^{\prime}(x y z) \leq \max \left\{f^{\prime}(x), f^{\prime}(y), f^{\prime}(z)\right\}$ for all $x, y, z \in T$.

(2) $f(x y z) \geq \min \{f(x), f(y), f(z)\}$ for all $x, y, z \in T$. 
Proof Straightforward.

Theorem 2.8 Let $f$ be a fuzzy subset of an ordered ternary semigroup $T$. Then $f$ is a fuzzy left filter (fuzzy right filter, fuzzy lateral filter, fuzzy filter) of $T$ if and only if the complement $f^{\prime}$ of $f$ is a prime fuzzy left ideal (prime fuzzy right ideal, prime fuzzy lateral ideal, prime fuzzy ideal) of $T$.

Proof Assume $f$ is a fuzzy left filter of $T$. Let $x, y \in T$ such that $x \leq y$. Since $f$ is a fuzzy left filter of $T, f(x) \leq f(y)$. This implies $f^{\prime}(x) \geq f^{\prime}(y)$. Next, let $x, y, z \in T$. Since $f$ is a fuzzy left filter of $T, f(x y z) \leq f(z)$. Thus $f^{\prime}(x y z) \geq f^{\prime}(z)$. Finally, let $x, y, z \in T$. Since $f$ is a fuzzy left filter of $T, f(x y z) \geq \min \{f(x), f(y), f(z)\}$. By Lemma $2.2, f^{\prime}(x y z) \leq$ $\max \left\{f^{\prime}(x), f^{\prime}(y), f^{\prime}(z)\right\}$.

Conversely, assume $f^{\prime}$ is a prime fuzzy left ideal of $T$. Let $x, y \in T$ such that $x \leq y$. Since $f^{\prime}$ is a fuzzy left ideal of $T, f^{\prime}(x) \geq f^{\prime}(y)$. Therefore $f(x) \leq f(y)$. Next, let $x, y, z \in T$. Since $f^{\prime}$ is prime, $f^{\prime}(x y z) \leq \max \left\{f^{\prime}(x), f^{\prime}(y), f^{\prime}(z)\right\}$. By Lemma 2.2, we have $f(x y z) \geq \min \{f(x), f(y), f(z)\}$. Finally, let $x, y, z \in T$. Since $f^{\prime}$ is a fuzzy left ideal of $T, f^{\prime}(x y z) \geq$ $f^{\prime}(z)$. Hence $f(x y z) \leq f(z)$.

The other parts can be proved in similarly way.

Corollary 2.1 Let $F$ be a nonempty subset of an ordered ternary semigroup $T$. Then $F$ is a left filter (right filter, lateral filter, filter) of $T$ if and only if the complement $f_{F}^{\prime}$ of $f_{F}$ is a prime fuzzy left ideal (fuzzy right filter, fuzzy lateral filter, fuzzy filter) of $T$.

Proof It follows by Theorem 2.2 and Theorem 2.8 .

Acknowledgment Most of the work in this paper is a part of the Master Thesis written by Mr.Sompob Saelee under the supervision of Assistant Professor Dr.Ronnason Chinram. Mr.Sompob Saelee would like to thank him for helpful suggestions.

\section{References}

Dixit, V. N. \& Dewan, S. (1995). A note on quasi and bi-ideals in ternary semigroups. Int. J. Math. Math. Sci., 18, 501-508.

Iampan, A. (2007). Lateral ideals of ternary semigroups. Ukrainian Math. Bull., 4, 517-526.

Kar, S. \& Maity, B. K. (2007). Congruences on ternary semigroups. J. Chugcheong Math. Soc., 20 , 191-200.

Kehayopulu, N. (1990). Remarks on ordered semigroups. Math. Japonica, 35, 1061-1063.

Kehayopulu, N. \& Tsingelis, M. (1999). A note on fuzzy sets in semigroups. Sci. Math., 2, 411-413.

Kehayopulu, N. \& Xiang-Yun Xie \& Tsingelis, M. (2001). A characterization of prime and semiprime ideals of semigroups in terms of fuzzy subsets. Soochow J. Math., 27, 139-144.

Kehayopulu, N. \& Tsingelis, M. (2002). Fuzzy sets in ordered groupoids. Semigroup Forum, 65, 128-132.

Kuroki, N. (1981). On fuzzy ideals and fuzzy bi-ideals in semigroups. Fuzzy Sets and Systems, 5, 203-215.

Kuroki, N. (1991). On fuzzy semigroups. Inf. Sci., 53, 203-236.

Kuroki, N. (1993). Fuzzy semiprime quasi-ideals in semigroups. Inf. Sci., 75, 201-211.

Lehmer, D. H. (1932). A ternary analoue of abelian groups, Amer. J. Math., 59, 329-338.

Los, J. (1955). On the extending of model I. Fundamenta Mathematicae, 42, 38-54.

Santiago, M. L. (1990). Regular ternary semigroups. Bull. Calcutta Math. Soc., 82, 67-71.

Shabir, M. \& Khan, A. (2008). Fuzzy filters in ordered semigroups. Lobachevskii J. Math., 29, 82-89.

Sioson, F. M. (1965). Ideal theory in ternary semigroups. Math. Japonica, 10, 63-84.

Zadeh, L. A. (1965). Fuzzy sets. Inf. Cont., 8, 338-353. 